\title{
Chronic Pelvic Pain in Women: Sharing Interdisciplinary Experience
}

\author{
Thomas Baptiste-Weiss, BSc'; Beatrice Cuzin, MSc, MD²* \\ 'Osteopathic Therapist in Integrative and Alternative Medicine, Geneva, Switzerland \\ ${ }^{2}$ Department of Urology and Transplantation, E Herriot University Hospital, Lyon Cedex 69437, France \\ "Corresponding author \\ Beatrice Cuzin, MSc, MD \\ Department of Urology and Transplantation, E Herriot University Hospital, Lyon Cedex 69437, France; Tél. 04 72 II II 40; E-mail: beatrice.cuzin@chu-lyon.fr
}

Article information

Received: September 26 ${ }^{\text {th }}$, 2021; Revised: December I ${ }^{\text {st }}, 2021$; Accepted: December 3 ${ }^{\text {rd }}, 2021$; Published: December $7^{\text {th }}$, 202I

\section{Cite this article}

Baptiste-Weiss T, Cuzin B. Chronic pelvic pain in women: Sharing interdisciplinary experience. Women Health Open J. 202I; 7(2): 3I-33. doi: 10.17I40/WHOJ-7-I44

\section{INTRODUCTION}

$\mathrm{C}$ hronic pelvic pain (CPP) is a common, burdensome, and costly condition that disproportionately affects women. Touching an estimated more than $20 \%$ of people worldwide or approximately 1 in 7 women, ${ }^{1}$ CPP is a common problem. In one study of reproductive-aged women in primary care practices, the reported prevalence rate of pelvic pain was $39 \%{ }^{2}$ Of all referrals to gynecologists, $10 \%$ are for pelvic pain. ${ }^{3}$

Ten percent $(10 \%)$ of the world's population - approximately 60 million people - endure chronic pain ${ }^{4}$ and fairly reliable estimates in individual countries and regions indicate chronic pain prevalence closer to $20-25 \% .{ }^{5,6}$ Primary care facilities in Asia, Africa, Europe, and in the Americas have patients reporting persistent pain prevalence of $10-25 \%$. Consistent estimates of chronic pain prevalence in the U.S. range from $12-25 \%$, and a prevalence of $20 \%$ has been noted in Europe. ${ }^{7}$ The World Health Organization (WHO) has estimated that as many as 1 in 10 adult individuals are newly diagnosed with chronic pain each year. ${ }^{8}$

Chronic pelvic pain differs from acute pelvic pain in several important ways. Acute pain typically arises from an inflammatory, infectious, or anoxic event or traumatic injury that resolves over time with treatment and recovery. When pain persists, a chronic stress phenotype may emerge and is characterized by a vicious cycle of physical and psychologic consequences. Prolonged activity restriction can lead to physical deconditioning. Continued fear, anxiety, and distress can lead to long-term psychosomatic deterioration and social isolation. Although mood symptoms are ubiquitous in chronic pain syndromes, criteria for major depression are met in approximately $12-33 \%$ of women across samples of women living with or seeking care for CPP.

Diagnosis and initial management of CPP in women are within the operative scope of specialists mainly in obstetrics and gynecology. In reality, the complexity of the muscular and osteopathic structures and pelvis innervation with the anatomical proximity of pelvic viscera means this condition frequently overlaps traditional medical specialties and could benefit from complementary medicine such as osteopathy, physiotherapy, or traditional Chinese medicine. A multidisciplinary approach which includes complementary and alternative medicine techniques in managing CPP in women seems to yield the best results. ${ }^{9}$ Although very few of these approaches have been evaluated in formal clinical trials so far, they have much to offer to those with chronic pain in terms of enhancing quality of life and pain-related coping, as well as reducing disability and pain-related interference with functioning.

\section{CHRONIC PELVIC PAIN}

Because CPP is often multifactorial, most patients will have multiple pain generators and comorbid conditions. Although many conditions are associated with CPP, determining how pain changes with sexual activity, menstruation, urination, and defecation is a good starting point. Patients often focus on visceral etiologies, yet neuromuscular issues such as myofascial trigger points may be more common and are often overlooked. ${ }^{9}$ Single-digit or swab palpation for tenderness of pelvic floor muscles, or palpation of the abdomen and the lower back, including the sacroiliac joints that generates pain can identify possible neuromuscular conditions. In one small study, pelvic floor muscle tenderness or a positive flexion, abduction, and external rotation test identified $85 \%$ of patients with chronic neuromuscular pelvic pain. Other clinical signs were statistically significantly associated with long-term pelvic chronic pain in women: these include suicidal risk, alcoholism, smoking, psychotropic drug use, psychosomatic and sleep disorders, post-traumatic stress disorder, vaginal discharge, pelvic pain, sexual disorders, diarrhea and chest pain.

A detailed medical history and physical examination, with particular attention to the abdominal and pelvic neuro musculo- 
skeletal examination, are recommended for the evaluation of CPP. Subtle physical sensitivity that increase the likelihood of neuro musculoskeletal contributors to CPP include pelvic floor muscle tenderness and abdominal wall tenderness that stimulate a reaction of pain in the patient.

Psychosocial factors play a role in all types of pain and can affect symptom severity and prognosis. Pelvic pain and dyspareunia are more prevalent in women with a history of abuse, mental illness, lack of support, social stressors, and relationship discord. These comorbidities do not alter the visceral or neuro musculoskeletal pain generators but may worsen the associated symptom burden and psychological effects. Treating psychosocial factors as separate but equally important pain contributors can increase the woman's awareness of her conscious and unconscious perception of pain and facilitate her recovery. Female genital mutilations should not be forgotten during the process of diagnosis and treatment.

As follows, CPP should receive greater attention as a global health care priority for women, particularly in developing countries. In order to be able to provide adequate, inexpensive care and treatment for this condition, pain management must be recognized as a human right, and access to this care for vulnerable populations must be a priority. All health care systems must engage in their obligation to provide this access to all women.

The non-exhaustive list of pelvic pain disorders encompasses many conditions: dyspareunia, cystalgia, bladder hyperexcitability, coccygodynia, dysmenorrhea and hypofertility, interstitial cystitis, vulvodynia, endometriosis, pelvic floor tension, sacral pain, lumbar pain, inter-discal pain, abdominal pain, with associated symptoms such as insomnia, headaches, nausea, and gastro-intestinal dysfunctions, trauma (e.g. secondary to vaginal delivery), surgery (e.g. any abdominal wall incision including caesarean section), pelvic floor muscle pain syndrome, vaginal muscle spasms, neuralgia from nerve entrapment or irritation, pain arising from the lower part of the spine (e.g. from sprains, strains, fractures, degenerative disease, disc lesions), sacroiliac joint dysfunction, symphysis pubis dysfunction, coccygeal pain, piriformis syndrome, myofascial pain syndrome, and abdominal migraines.

\section{RECOMMENDATIONS}

The main recommendations for the management of pelvic pain vary from woman to woman depending on her personal life story. Recommendations are often based on listening to and understanding the needs of women with this complex condition. The time required to treat these women varies according to the time spent on clinical evaluation and the means available to physicians for their treatment. The cost of the consultation also rests a choice to be made regarding the spectrum of the general clinical evaluation and the orientations granted according to the location of the pathology. The total cost of care will differ from one country to another depending on the level of training of the caregivers and the treatments available. Practical assessment of pain levels measured by type, myofascial pain, medications and surgical interven- tions, principles of opioid management, increased use of magnetic resonance imaging (MRI), documentation of the extent of disease seen through surgery, non-conventional therapies (acupuncture, osteopathy, pelvic reducation through electrostimulation), access to multidisciplinary models of care with components of physiotherapy, posturology, psychological and psychiatric care for trauma, in conjunction with other medical disciplines, such as gynecology and anesthesia also play a significant role in the causal orientation of this type of pathology.

\section{CONCLUSION}

The organization Formations Sans Frontières-International Health Care Education ${ }^{10}$ has been working since 2004 to sensitize health authorities as well as national and local associations to promote training in sexual and reproductive health.

Considering broad measures in favor of the creation of multidisciplinary teams through advanced training of health professionals in its sectors strengthens local capacities to ensure the management of this pathology.

The evidence-based literature on the treatment of CPP remains limited. The majority of treatments are too often focused on symptomatic pain relief, due to a lack of multidisciplinary working capacity of health care providers and financial resources for training and treatment. The approach to the treatment of chronic pelvic diseases should be focused on the pathology and the history of the person, taking into consideration the co-morbidity factors of the individual in their medical history and life circumstances. If, however, the origin of the pelvic pain remains unknown, it is recommended that the patient undergo further evaluation with alternative approaches such as osteopathy, traditional Chinese medicine, yoga, breathing exercises, hypnosis, and eye movement desensitization and reprocessing (EMDR) - especially if the case may be traumatic, following rape and/or abusive touching that is often perpetuated (and/or unspoken) during medical consultations.

Multidisciplinary management of chronic pain should be offered to women with CPP within the public health care system of each country. The strengthening of training programs for health care providers should be systematically considered in health care centers and should systematically include a program in medical and paramedical universities. Raising women's awareness from an early age through sex education campaigns in school programs is also essential to increasing early detection and improving treatment of this condition and to apply preventive measures such as training in posture, hygiene, exercise and breathing.

\section{CONFLICTS OF INTEREST}

The authors declare that they have no conflicts of interest.

\section{REFERENCES}

1. Mathias SD, Kuppermann M, Liberman RF, Lipschutz RC, Stee- 
ge JF. Chronic pelvic pain: prevalence, health-related quality of life, and economic correlates. Obstet Gynecol. 1996; 87(3): 321-327 doi: 10.1016/0029-7844(95)00458-0

2. Jamieson DJ, Steege JF. The prevalence of dysmenorrhea, dyspareunia, pelvic pain, and irritable bowel syndrome in primary care practices. Obstet Gynecol. 1996; 87(1): 55-58. doi: 10.1016/00297844(95)00360-6

3. Reiter, RC. A profile of women with chronic pelvic pain. Clin Obstet Gynecol. 1990; 33(1): 130-136.

4. Breivik H. International association for the study of pain. Journal of Pain and Symptom Management. 2002; 24(2): 97-101. doi: 10.1016/ S0885-3924(02)00465-7

5. Gureje O, Von Korff M, Simon GE, Gater R. Persistent pain and well-being: A World Health Organization study in primary care. JAMA. 1998; 280(2): 147-151. doi: 10.1001/jama.280.2.147

6. Gureje O, Von Korff M, Simon GE, Gater R. Executive Sum- mary and Highlights. 2006. Web site. http://www.cdc.gov/nchs/ data/hus/hus06.pdf. Accessed September 25, 2021.

7. Breivik H, Collett B, Ventafridda V, Cohen R, Gallacher D. Survey of chronic pain in Europe: prevalence, impact on daily life, and treatment. Eur J Pain. 2006; 10(4): 287-333. doi: 10.1016/j. ejpain.2005.06.009

8. International Association for the Study of Pain. Unrelieved pain is a major global healthcare problem. Web site. http://www.iasppain.org $/ \mathrm{AM} /$ Template.cfm?Section $=$ Home\&Template $=/ \mathrm{CM} /$ ContentDisplay.cfm\&ContentID $=2908$. Accessed September 25, 2021.

9. No authors listed. Chronic pelvic pain: ACOG practice bulletin, Number 218. Obstet Gynecol. 2020; 135(3): e98-e109. doi: 10.1097/ AOG.0000000000003716

10. Our Work Promise To Uphold The Trust Placed. Web site. www.fsf-ihce.africa. Accessed September 25, 2021. 